Original Research Paper

\title{
A New Spectral Conjugate Gradient Method for Nonlinear Unconstrained Optimization
}

\author{
${ }^{1}$ Ibtisam A. Masmali, ${ }^{2 *}$ Zabidin Salleh and ${ }^{3}$ Ahmad Alhawarat \\ ${ }^{1}$ Department of Mathematics, Faculty of Science, Jazan University, Jazan, Saudi Arabia \\ ${ }^{2}$ Department of Mathematics, Faculty of Ocean Engineering Technology and Informatics, \\ University Malaysia Terengganu, 21030 Kuala Nerus, Terengganu, Malaysia \\ ${ }^{3}$ Department of Mathematics, Faculty of Ocean Engineering Technology and Informatics, \\ University Malaysia Terengganu, 21030 Kuala Nerus, Terengganu, Malaysia
}

\author{
Article history \\ Received: 13-03-2021 \\ Revised: 17-06-2021 \\ Accepted: 25-06-2021 \\ Corresponding Author: \\ Zabidin Salleh \\ Department of Mathematics, \\ Faculty of Ocean Engineering \\ Technology and Informatics, \\ Universiti Malaysia \\ Terengganu, 21030 Kuala \\ Nerus, Terengganu, Malaysia \\ Email: zabidin@umt.edu.my
}

\begin{abstract}
The conjugate gradient method is widely used to solve large scale unconstrained optimization problems. However, the rate of convergence conjugate gradient method is linear unless it restarted. In this study, we present a new spectral conjugate gradient modification formula with restart property obtains the global convergence and descent properties. In addition, we proposed a new restart condition for Fletcher-Reeves conjugate gradient formula. The numerical results demonstrated that the modified Fletcher-Reeves parameter and the new CG formula with their restart conditions are more efficient and robustness than other conventional methods.
\end{abstract}

Keywords: Conjugate Gradient, Global Convergence, Descent Condition

\section{Introduction}

We consider the following problem:

$\min f(x), x \in R^{n}$,

where, $f: R^{n} \rightarrow R$ is continuous and differentiable function and its gradient $g(x)=\Delta f(x)$ is available. Iterative methods are usually used to solve (1), as follows:

$x_{k+1}=x_{k}+\alpha_{k} d_{k}, k=1,2, \ldots$,

starting from initial point $x_{1} \in R^{n}$, where $\alpha_{k}$ is obtained by some line search. The search direction $d_{k}$ is defined by:

$d_{k}=\left\{\begin{array}{l}-g_{k}, k=1, \\ -g_{k}+\beta_{k} d_{k-1}, k \geq 2,\end{array}\right.$

where, $g_{k}=g\left(x_{k}\right)$ and $\beta_{k}$ is known as the conjugate gradient parameter.

The exact line search can be used to find the steplength $\alpha_{k}$. Suppose that $\phi(\alpha)=f\left(x_{k}+\alpha d_{k}\right)$ which is problem that departs from $x_{k}$ to find a step length in the direction $d_{k}$ such that $\phi(\alpha)<\phi(0)$. If the step length is defined such that the search direction minimized i.e., this line search is called exact line search where this line search is expensive.
Therefore, using the inexact line search with less computation load is better. The inexact line search in particular Strong Wolfe-Powell (SWP) line search inherits the advantages of exact line search and computationally inexpensive. Thus, to reduce the computation cost of exact line search and also to reduce evaluations of the objective function and gradient function, usually the inexact line search is employed. SWP line search is more preferable than other line searches. The SWP line search is defined by:

$$
\begin{aligned}
& f\left(x_{k}+\alpha_{k} d_{k}\right)=\min f\left(x_{k}+\alpha d_{k}\right), \alpha \geq 0, \\
& f\left(x_{k}+\alpha_{k} d_{k}\right) \leq f\left(x_{k}\right)+\delta \alpha_{k} g_{k}^{T} d_{k}
\end{aligned}
$$

and:

$\left|g\left(x_{k}+\alpha_{k} d_{k}\right)^{T} d_{k}\right| \leq \sigma\left|g_{k}^{T} d_{k}\right|$

where, $0<\delta<\sigma<1$. The Weak Wolfe-Powell (WWP) (Wolfe, 1969; 1971) line search given by (5) and:

$$
g\left(x_{k}+\alpha_{k} d_{k}\right)^{T} d_{k} \geq \sigma g_{k}^{T} d_{k} .
$$

The convergence of CG method will not be linear if we restart CG method (Powell, 1977). Beale (1972) 
recommended the use of the two-term CG method instead $\left(d_{k}=-g_{k}, \forall k \geq 1\right)$ as the restart search direction. Powell (1984) recommended restarting $d_{k}$ using Beale's method if:

$$
\left|g_{k}^{T} g_{k-1}\right|>0.2\left\|g_{k}\right\|^{2}
$$

Dai and Yuan (1998) present the following restart criterion:

$$
\left|g_{k}^{T} g_{k-1}\right|>\tau\left\|g_{k}\right\|^{2}, \tau \in(0,1)
$$

The famous formulas for $\beta_{k}$ are the Hestenes-Stiefel (HS) (Hestenes and Stiefel, 1952), Fletcher-Reeves (FR) (Fletcher and Reeves, 1964) and Polak-Ribière-Polyak (PRP) (Polak and Ribiere, 1969) formulas, which are defined as follows:

$$
\beta_{k}^{H S}=\frac{g_{k}^{T} y_{k}}{d_{k-1}^{T} y_{k}} \beta_{k}^{F R}=\frac{g_{k}^{T} g_{k}}{\left\|g_{k-1}\right\|^{2}} \beta_{k}^{P R P}=\frac{g_{k}^{T} y_{k}}{\left\|g_{k-1}\right\|^{2}},
$$

where, $y_{k}=g_{k}-g_{k-1}$.

Polak and Ribière (1969) proved CG method with the PRP formula and by using exact line search is convergent. Powell (1984) show that the PRP fail to satisfy the convergence by using an example even the exact line is used. Powell recommended to use the non-negative of PRP formula to satisfy the convergence analysis. Gilbert and Nocedal (1992) suggest to use PRP as follows:

$$
\beta_{k}^{P R P+}=\max \left\{0, \beta_{k}^{P R P}\right\}
$$

Zoutendijk (1970) obtain the global convergence of FR formula with CG method and the exact line search. Al-Baali (1985) proved FR method with SWP line search when $\sigma<1 / 2$ and SWP line search is employed, Guanghui et al. (1995) extended the proof to the case for $\sigma \leq 1 / 2$.

Alhawarat et al. (2017) presented the following formula:

$$
\beta_{k}^{A Z P R P}=\left\{\begin{array}{l}
\frac{\left\|g_{k}\right\|^{2}-\mu_{k}\left|g_{k}^{T} g_{k-1}\right|}{\left\|g_{k-1}\right\|^{2}}, \text { if } \quad\left\|g_{k}\right\|^{2}>\mu_{k}\left|g_{k}^{T} g_{k-1}\right|, \\
0, \text { otherwise, }
\end{array}\right.
$$

where, $\mu_{k}$ is defined as follows:

$$
\mu_{k}=\frac{\left\|x_{k}-x_{k-1}\right\|}{\left\|y_{k}\right\|}
$$

Kaelo et al. (2020) proposed the following CG formula:

$$
\beta_{k}^{P K T}=\left\{\begin{array}{l}
\frac{\left\|g_{k}\right\|^{2}-g_{k}^{T} g_{k-1}}{\max \left\{d_{k-1}^{T} y_{k-1},-g_{k-1}^{T} d_{k-1}\right\}}, \text { If } 0<g_{k}^{T} g_{k-1}<\left\|g_{k}\right\|^{2} \\
\frac{\left\|g_{k}\right\|^{2}}{\max \left\{d_{k-1}^{T} y_{k-1},-g_{k-1}^{T} d_{k-1}\right\}}, \text { otherwise. }
\end{array}\right.
$$

As we know that in the case of the function is quadratic i.e., $f(x)=g^{T} x+(1 / 2) x^{T} H x$ and the step size obtained by exact line search (3), the CG method satisfy the conjugacy condition i.e., $d_{i}^{T} H d_{j}^{T}=0, \forall i \neq j$. By using the mean value theorem and exact line search with Eq. (2) we can obtain $\beta_{k}^{H S}$ . From quasi-Newton method, BFGS method and the limited memory (LBFGS) method and using (2), Dai and Liao (2001) present the following conjugacy condition:

$$
d_{k}^{T} y_{k-1}=-\operatorname{tg}_{k}^{T} s_{k-1}
$$

where, $S_{k-1}=x_{k}-x_{k-1}$ and $t \geq 0$. In the case of $t=0 \mathrm{Eq}$. (8) becomes the classical conjugacy condition. By using (2) and (8), (Kaelo et al., 2020) proposed the following $\mathrm{CG}$ formula:

$$
\beta_{k}^{D L}=\frac{g_{k}^{T} y_{k-1}}{d_{k-1}^{T} y_{k-1}}-t \frac{g_{k}^{T} s_{k-1}}{d_{k-1}^{T} y_{k-1}} .
$$

However, $\beta_{k}^{D L}$ face the same problem as $\beta_{k}^{P R P}$ and $\beta_{k}^{H S}$ i.e., $\beta_{k}^{D L}$ is not non-negative in general. Thus (Dai and Liao, 2001) replaced Eq. (9) by:

$$
\beta_{k}^{D L+}=\max \left\{\beta_{k}^{H S}, 0\right\}-t \frac{g_{k}^{T} s_{k-1}}{d_{k-1}^{T} y_{k-1}} .
$$

Hager and Zhang $(2005 ; 2013)$ presented a modified CG parameter that satisfies the descent property for any inexact line search with $g_{k}^{T} d_{k} \leq-(7 / 8)\left\|g_{k}\right\|^{2}$. This formula is given as follows:

$$
\beta_{k}^{H Z}=\max \left\{\beta_{k}^{N}, \eta_{k}\right\}
$$

where,

$$
\beta_{k}^{N}=\frac{1}{d_{k}^{T} y_{k}}\left(y_{k}-2 d_{k} \frac{\left\|y_{k}\right\|^{2}}{d_{k}^{T} y_{k}}\right)^{T} g_{k},
$$
$\eta_{k}=-\frac{1}{\left\|d_{k}\right\| \min \left\{\eta,\left\|g_{k}\right\|\right\}}$ and $\eta>0$ is a constant. Notes that if $t=2 \frac{\left\|y_{k}\right\|^{2}}{s_{k}^{T} y_{k}}$ then $\beta_{k}^{N}=\beta_{k}^{D Y}$.

The positive scalar denoted by $\theta_{k}$. Hence, $d_{k}$ given as:

$$
d_{k}=-\theta_{k} g_{k}+\beta_{k} d_{k-1} \text {. }
$$


when, $\theta_{k}=1$, the search direction is a classical CG method. If $\beta_{k}=0$, then there are two possibilities of $\theta_{k}$. If $\theta_{k}=\Delta^{2} \mathrm{f}\left(x_{k}\right)^{-1}$ or an approximation of it, then the search direction is Newton or Quasi-Newton, respectively.

\section{The New Formula and the Algorithm}

Here, we construct the following new modification to improve the efficiency and robustness of DY CG formula and robustness of PRP CG method.

For $k=1, d k=-g_{k}$.

For $k \geq 2$ :

$d_{k}^{A T A Z}=\left\{\begin{array}{l}-\theta_{k} g_{k}+\beta_{k}^{D Y} d_{k-1}, \text { if } g_{k}^{T} d_{k-1} \geq 0, \\ -g_{k}+\beta_{k}^{P R P+} d_{k-1}, \text { else. }\end{array}\right.$

where, $\|$.$\| means the Euclidean norm and$ $\theta_{k}=1+\frac{g_{k}^{T} d_{k-1}}{g_{k-1}^{T} d_{k-1}}$ and:

$$
\beta_{k}^{D Y}=\frac{\left\|g_{k}\right\|^{2}}{d_{k-1}^{T}\left(g_{k}-g_{k-1}\right)} .
$$

\section{Algorithm 1}

Step 1 Provide a starting point $x_{1}$. Set the initial search direction $d_{1}=-g_{1}$. Let $k:=1$.

Step 2 If a stopping criteria is satisfied, then stop.

Step 3 Compute $d_{k}$ based on (2) with (11).Error!

Reference source not found.

Step 4 Compute $\alpha_{k}$ using (4) and (6).

Step 5 Update $x_{k+1}$ based on (1).

Step 6 Set $k:=k+1$ and go to Step 2 .

In Algorithm1, note that after the step $k=k+1$, the iterates $x_{k}=x_{k+1}$ takes place after every iteration. The other iterations are updated in a similar manner as $x_{k}$.

In following section, we present the global convergence property of the new formula (11). In case of $d_{k}^{A T R Z}=0$, then the search direction becomes the steepest descent (negative gradient) which mean the stationary point is obtained.

\section{Convergence of CG Algorithm with the Search Direction $d_{k}^{A T A Z}$}

\section{Assumption 1}

A. The level set $\Omega=\left\{x \mid f(x) \leq f\left(x_{1}\right)\right\}$ is bounded, that is, a positive constant $M$ exists such that

$$
\|x\| \leq M, \forall x \in \Omega .
$$

B. In some neighbourhood $n$ of $\Omega, f$ is continuously differentiable and its gradient is Lipschitz continuous; that is, for all $x, y \in N$, there exists a constant such that:

$$
\|g(x)-g(y)\| \leq L\|x-y\| .
$$

This assumption implies that there exists a positive constant $B$ such that:

$$
\|g(u)\| \leq B, \forall u \in N
$$

The descent condition:

$$
g_{k}^{T} d_{k} \leq-\left\|g_{k}\right\|^{2}, \forall k \geq 1,
$$

Al-Baali (1985) modified (12) to the following form and used it to prove the FR method:

$g_{k}^{T} d_{k} \leq-c\left\|g_{k}\right\|^{2}, \forall k \geq 1$,

where, $c \in(0,1)$. Equation (13) is the sufficient descent condition. Note that the general form of the sufficient descent condition is (14) with $c>0$.

Descent and Convergence Properties for $d_{k}^{A T A Z}$ with the SWP Line Search

In fact, we have two types of global convergence; weak global convergence and strong global convergence both of them imply the stationary point for optimization problem. However, the convergence and the descent properties will not give any sense in terms of the efficiency for CG methods; for example, FR formula has global convergence properties with poor efficiency. Thus, to improve the efficiency when the method cycle does not reach a solution the CG algorithm should be restarted. In the following section, we will present a new CG method with restart property by using the steepest descent method.

The following lemma is called Zoutendijk condition (Al-Baali, 1985).

\section{Lemma 3.1}

Suppose assumption 1 is holds. Suppose method in the form (1), (2) and $\alpha_{k}$ satisfies the WWP line search (5) and (6), where the search direction satisfied. Then

$\sum_{k=0}^{\infty} \frac{\left(g_{k}^{T} d_{k}\right)^{2}}{\left\|d_{k}\right\|^{2}}<\infty$.

Also we can extended Eq. (14) to the following form:

$\sum_{k=0}^{\infty} \frac{\left\|g_{k}\right\|^{4}}{\left\|d_{k}\right\|^{2}}<\infty$. 
Kaelo et al. (2020) present the following theorem for global convergence properties:

\section{Theorem 3.1}

Let assumption 1 holds. Suppose any CG method in the form (1) and (2), where $d_{k}$ is a descent direction and $\alpha_{k}$ is obtained by the SWP line search. If:

$$
\sum_{k \geq 1}^{\infty} \frac{1}{\left\|d_{k}\right\|^{2}}=\infty
$$

then:

$$
\liminf _{k \rightarrow \infty}\left\|g_{k}\right\|=0
$$

\section{Theorem 3.2}

Let the sequence $\left\{g_{k}\right\}$ and $\left\{d_{k}\right\}$ are generated by the methods (2), (3) and (11), then (13) holds.

Proof. By using proof by induction. From (3) for $k=$ $1, g_{1}^{T} d_{1}=-\left\|g_{1}\right\|^{2}$. Suppose that it is true until $k$ - 1, i.e., $g_{i-1}^{T} d_{i-1}<0$, for $i=1,2, \ldots, k-1$ then we have the following two cases:

Case $1 g_{k}^{T} d_{k-1} \geq 0$ :

$$
d_{k}=-\frac{g_{k}^{T} d_{k-1}}{g_{k-1}^{T} d_{k-1}} g_{k}-g_{k}+\frac{\left\|g_{k}\right\|^{2}}{d_{k-1}\left(g_{k}-g_{k-1}\right)} d_{k-1},
$$

Multiply both sides by $g_{k}^{T}$ :

$g_{k}^{T} d_{k}=-\frac{g_{k}^{T} d_{k-1}}{d_{k-1}\left(g_{k}-g_{k-1}\right)}\left\|g_{k}\right\|^{2}-\left\|g_{k}\right\|^{2}+\frac{\left\|g_{k}\right\|^{2}}{d_{k-1}\left(g_{k}-g_{k-1}\right)} g_{k}^{T} d_{k-1}$,

Since $g_{k}^{T} d_{k-1} \geq 0$ :

$$
g_{k}^{T} d_{k}=-\left\|g_{k}\right\|^{2}
$$

Case $2 g_{k}^{T} d_{k-1}<0$ :

$$
d_{k}=-g_{k}+\beta_{k}^{P R P+} d_{k-1},
$$

Multiply both sides by $g_{k}^{T}$ :

$$
g_{k}^{T} d_{k}=-\left\|g_{k}\right\|^{2}+\beta_{k}^{P R P+} g_{k}^{T} d_{k-1},
$$

By using $g_{k}^{T} d_{k-1}<0$ and $\beta_{k}^{P R P+} \geq 0$, we obtain $g_{k}^{T} d_{k}<0$.

\section{Theorem 3.3}

Let assumption 1 holds. Assume $\left\{g_{k}\right\}$ and $\left\{d_{k}\right\}$ are obtained by algorithm 1 in which $\alpha_{k}$ is obtained by the WWP line search and (13) holds. Then the $\liminf _{k \rightarrow \infty}\left\|g_{k}\right\|=0$.

$$
\begin{aligned}
& d_{k}=-g_{k}+\frac{g_{k}^{T} d_{k-1}}{g_{k-1}^{T} d_{k-1}} g_{k}+\frac{\left\|g_{k}\right\|^{2}}{d_{k-1}\left(g_{k}-g_{k-1}\right)} d_{k-1}, \\
& \left\|d_{k}\right\|=\left\|\theta_{k}\right\|\|g\|_{k}+\frac{\left\|g_{k}\right\|^{2}}{\left\|d_{k-1}\right\|\left\|\left(g_{k}-g_{k-1}\right)\right\|}\left\|d_{k-1}\right\| \\
& \left\|d_{k}\right\|=\left\|\theta_{k}\right\|\|g\|_{k}+\frac{\left\|g_{k}\right\|^{2}}{\left\|\left(g_{k}-g_{k-1}\right)\right\|}
\end{aligned}
$$

since $\left\|\theta_{k}\right\| \leq 1-\sigma$

$$
\left\|d_{k}\right\|=\|g\|_{k}-\sigma\|g\|_{k}+\frac{\left\|g_{k}\right\|^{2}}{\left\|\left(g_{k}-g_{k-1}\right)\right\|}
$$

By using assumption 1:

$$
\left\|d_{k}\right\|=1-\sigma \gamma+\frac{\gamma^{2}}{\lambda} \text { where } \lambda>0
$$

Let: $M=1-\sigma \gamma+\frac{\gamma^{2}}{\lambda}\left\|d_{k}\right\| \leq M$.

By using Theorem 3.1 We obtain the $\lim _{k \rightarrow \infty} \inf \left\|g_{k}\right\|=0$.

\section{The New Restart Criteria for FR Family}

Fletcher-Reeves formula is simple CG method and has a global convergence property with SWP line search and it satisfies the descent property. However, FR formula is not efficient as $\beta_{k}^{P R P}$ which the later has a problem in convergence properties for some optimization functions. Powell studied $\beta_{k}^{F R}$ formula and show that this method cycle does not reach a solution when $x_{k+1} \approx x_{k}$ which implies that $\left\|g_{k}\right\| /\left\|g_{k-1}\right\| \approx 1$. To solve this problem we suggest restarting $\beta_{k}^{F R}$ as follows:

$\beta_{k}^{F R^{*}}=\left\{\begin{array}{l}0, \quad 0.9 \leq \frac{\left\|g_{k}\right\|}{\left\|g_{k-1}\right\|} \leq 1.1, \\ \beta_{k}^{F R}, \text { otherwise. }\end{array}\right.$

It is clear that when $\beta_{k}^{F R^{*}}$ will restart when the $\beta_{k}^{F R} \approx 1$.

\section{Numerical Results and Discussion}

To study the efficiency of the new search direction, we selected several test problems in Table 1 from Cuter (Bongartz et al., 1995) and Andrei (2008). The test functions consist of unimodal and multimodal functions. We also selected examples according to the similarities in significant physical properties and shapes. For example, the Rosenbrock function has a long, narrow shape; the Himmelblau function, the six-hump function and the three-hump function have many local minima; the Booth 
function is plate shaped; and the Sum Squares function is bowl shaped. As the CG method is useful for small-and large-scale optimisation problems, we also select the dimensions of the functions, which varied from 2 to 10000. All of the functions are nonlinear. In Table 1, "Gen" denotes generalised, "Ext" denotes extended, "Dim" denotes dimension/s.

We employed the MATLAB programming environment (ver. 7.9). The results are shown in Fig. 1 and 2 , in which a performance measure introduced by Dolan and More (2002) was employed.

The comparison include PRP+, FR, $d_{k}^{A T A Z}$ and FR* methods. $\left\|g_{k}\right\| \leq 10^{-6}$ is used as the stopping criteria for all algorithms. To obtain the step length we used strong Wolfe-Powell line search with $\delta=0.01$ and $\sigma=0.1$.

Since we are interested to find the stationary point/s for optimization problems, we selected more than one initial point to test every function in Table 1 the dimension of functions between 2 and 5000. Different initial points almost will obtain different stationary points, which imply that more than one solution for multimodal functions. Hence, we obtain the best solution. In addition, we select small and large dimensions for every function. The ranges of dimensions are chosen between 2 and 10000. Thus, we conclude that using different dimension and different initial points to obtain the results will be more convince than using original initials and one dimension. However, the starting point needs more study.
Figure 1 and 2 show that the curve of the new formula $\left(\beta_{k}^{A T A Z}\right)$ is uppermost of all curves. In addition, it is clear that $\mathrm{FR}^{*}$ formula is better than original FR formula which demonstrates the discussion that presented by Powell and the program is terminated by the user when the number of iterations exceeds 1000 . The PRP + formula is efficient since its curve started uppermost other curves. However, it is not satisfied the descent property with SWP line search. Thus, the program is terminated automatically.

In addition we present the following two functions the first one called Extended Beale function which given by the following formula:

$$
\begin{aligned}
& f(x)=\sum_{i=1}^{n / 2}\left(1.5-x_{2 i-1}\left(1-x_{2 i}\right)\right)^{2} \\
& +\left(2.25-x_{2 i-1}\left(1-x_{2 i}^{2}\right)\right)^{2}+\left(2.625-x_{2 i-1}\left(1-x_{2 i}^{3}\right)\right)^{2}
\end{aligned}
$$

Number of variables (n): 500, 1000, 5000, 10000 with initial points: $(-1,-1, \ldots,-1),(.5, .5, \ldots, .5),(1$, $1, \ldots, 1),(2,2, \ldots, 2)$.

This function has only one global minimum surrounded by a flat plateau. At the four corners lie four ascending steep walls that become smaller at the tip. These steep walls become higher as the value of the two variables increases. The minimum is $x^{*}=(3,0.5)$ and the function value is $f\left(x^{*}\right)=0$ for two variable functions (note Fig. 3 for a three-dimensional graph).

Table 1: A list of test functions

\begin{tabular}{ll}
\hline Function & Initial points \\
\hline Ext. White \& Holst function, & $(-1.2,1,-1.2,1 \ldots),(5,5, \ldots, 5),(10,10, \ldots, 10),(15,15, \ldots .15)$ \\
Ext. Rosenbrock function, & $(-1.2,1,-1.2,1 \ldots),(5,5, \ldots, 5),(10,10, \ldots, 10),(15,15, \ldots 15)$ \\
Six hump function & $(1,1),(5,5),(10,10),(15,15)$ \\
Ex. Beale function, & $(-1,-1, \ldots,-1),(.5,5, \ldots, 5),(1,1 \ldots, 1),(2,2, \ldots, 5),(5,5, \ldots, 5)$ \\
Three hump function & $(1,1),(5,5),(10,10),(15,15)$ \\
Ext. Himmelblau function & $(1,1, \ldots, 1),(5,5, \ldots, 5),(10,10 \ldots, 10),(15,15, \ldots, 15)$ \\
Diagonal 2 function & $(0.2,0.2, \ldots, 0.2),(0.25,0.25, \ldots, 0.25),(0.5, \ldots, 0.5),(1,1, \ldots, 1)$ \\
NONSCOMP function & $(1,1, \ldots, 1),(-1,-1, \ldots,-1),(-2,-2 \ldots,-2),(-5,-5, \ldots,-5)$ \\
Ext. DENSCHNB function & $(1,1, \ldots, 1),(5,5, \ldots, 5),(10,10 \ldots, 10),(15,15, \ldots, 15)$ \\
Shallow function & $(-2,-2, \ldots,-2),(2,2, \ldots, 2),(5,5 \ldots, 5),(10,10, \ldots, 10)$ \\
Booth function & $(1,1),(5,5),(10,10),(15,15)$ \\
DIXMAANA function, 26$]$ & $(2,2, \ldots, .2),(5,5, \ldots, 5),(10,10 \ldots, 10),(15,15, \ldots, 15)$ \\
DIXMAANB function & $(-2,-2, \ldots,-2),(-1,-1, \ldots,-1),(0,0 \ldots, 0),(1,1, \ldots, 1)$ \\
NONDIA function & $(-2,-2, \ldots,-2),(-1,-1, \ldots,-1),(0,0 \ldots, 0),(1,1, \ldots, 1)$ \\
Ext. Tridiagonal 1 function & $(1,1, \ldots, 1),(5,5, \ldots, 5),(10,10 \ldots, 10),(15,15, \ldots, 15)$ \\
DQDRTIC function & $(-1,-1, \ldots,-1),(1,1, \ldots, 1),(2,2 \ldots, 2),(3,3, \ldots, 3)$ \\
Diagonal 4 function & $(1,1, \ldots, 1),(5,5, \ldots, 5),(10,10 \ldots, 10),(15,15, \ldots, 15)$ \\
Raydan 2 function & $(1,1, \ldots, 1),(5,5, \ldots, 5),(10,10 \ldots, 10),(15,15, \ldots, 15)$ \\
Ext. DENSCHNB function & $(1,1, \ldots, 1),(5,5, \ldots, 5),(10,10 \ldots, 10),(15,15, \ldots, 15)$ \\
A Quadratic function QF2 & $(1,1, \ldots, 1),(5,5, \ldots, 5),(10,10 \ldots, 10),(15,15, \ldots, 15)$ \\
Zettl function & $(1,1, \ldots, 1),(5,5, \ldots, 5),(10,10 \ldots, 10),(15,15, \ldots, 15)$ \\
Extended Cliff & $(1,1, \ldots, 1),(5,5, \ldots, 5),(10,10 \ldots, 10),(15,15, \ldots, 15)$ \\
Ext. Powell function & $(1,1, \ldots, 1),(5,5, \ldots, 5),(10,10 \ldots, 10),(15,15, \ldots, 15)$ \\
Generalized Quartic GQ1 function & $(1,1, \ldots, 1),(5,5, \ldots, 5),(10,10 \ldots, 10),(15,15, \ldots, 15)$ \\
Ext. Block Diagonal BD1 function & $(1,1, \ldots, 1),(5,5, \ldots, 5),(10,10 \ldots, 10),(15,15, \ldots, 15)$ \\
\hline &
\end{tabular}


Ibtisam A. Masmali et al. / Journal of Computer Science 2021, 17 (6): 598.609 DOI: $10.3844 /$ jessp.2021.598.609

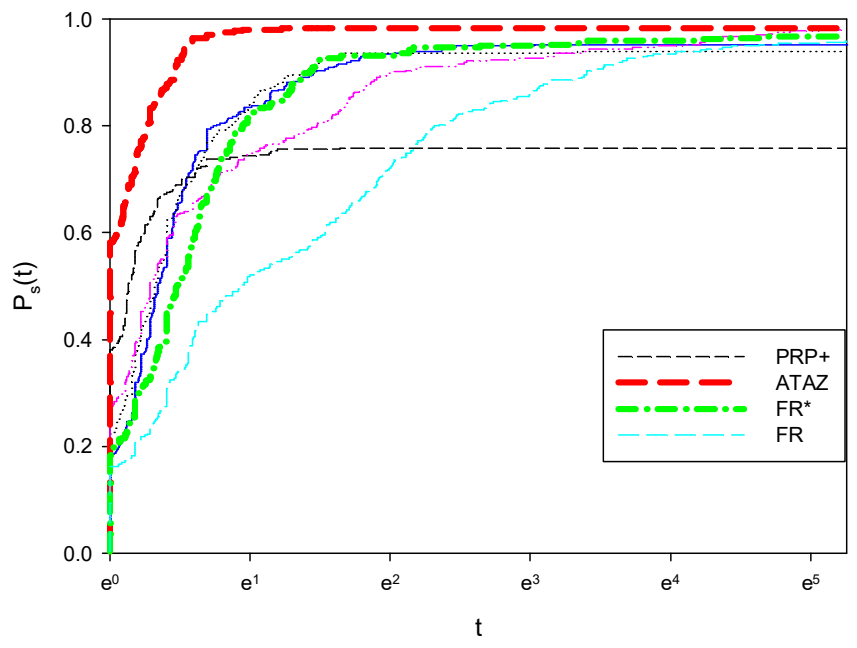

Fig. 1: Performance profile based on the number of iteration

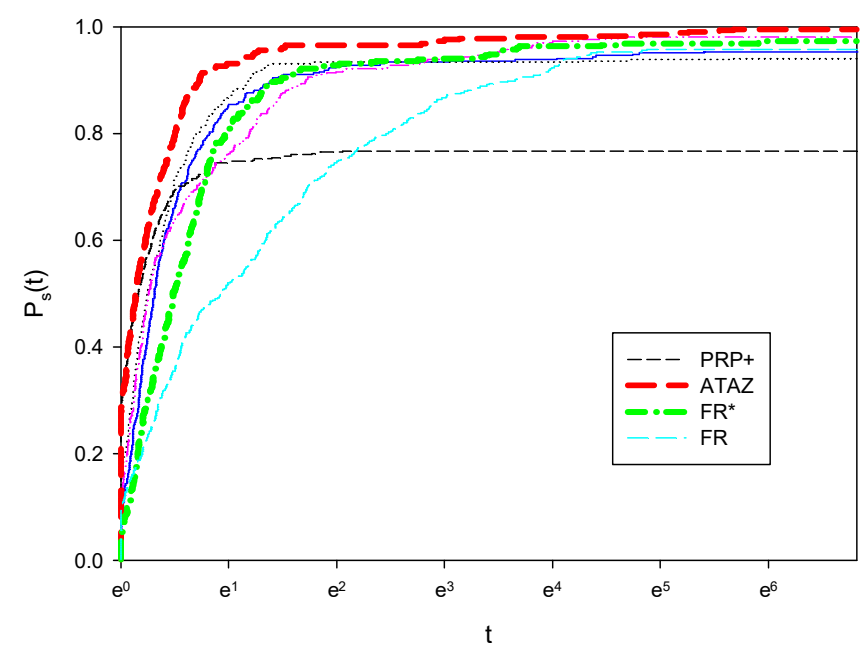

Fig. 2: Performance profile based on the CPU time

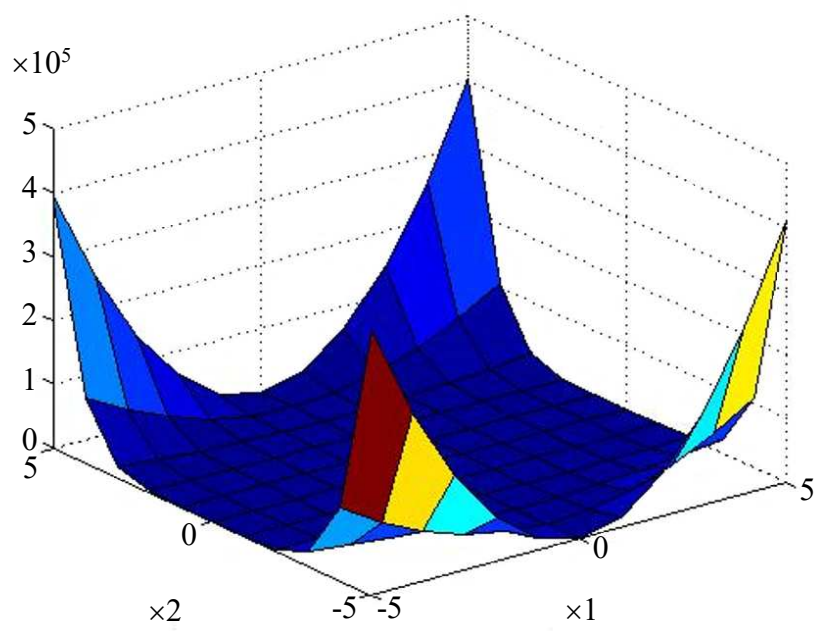

Fig. 3: Extended Beale function in 3D 
And the second function called

Perturbed quadratic function:

$f(x)=\left(\sum_{i=1}^{n} x_{i}\right)^{2}+\sum_{i=1}^{n} \frac{i}{100} x_{i}^{2}$

Initial points: $(0.5,0.5, \ldots, 0.5)$

This function has a smooth curve, look like dish shape and has a minimum value at $x^{*}=(0,0)$ and the function value is $f\left(x^{*}\right)=0$. where lies at the bottom for two variable functions (Fig. 4 for a three-dimensional graph).

Moreover we present another strong comparison between ATAZ and CG-Descent is given with benchmark functions in Table 2. The numerical results in Fig. 5, 6 and 7 show that the new modification ATAZ is better than CG-Descent in term of number of iterations, number of function evaluations and CPU time. The test functions can be downloaded from (Bongartz et al., 1995).

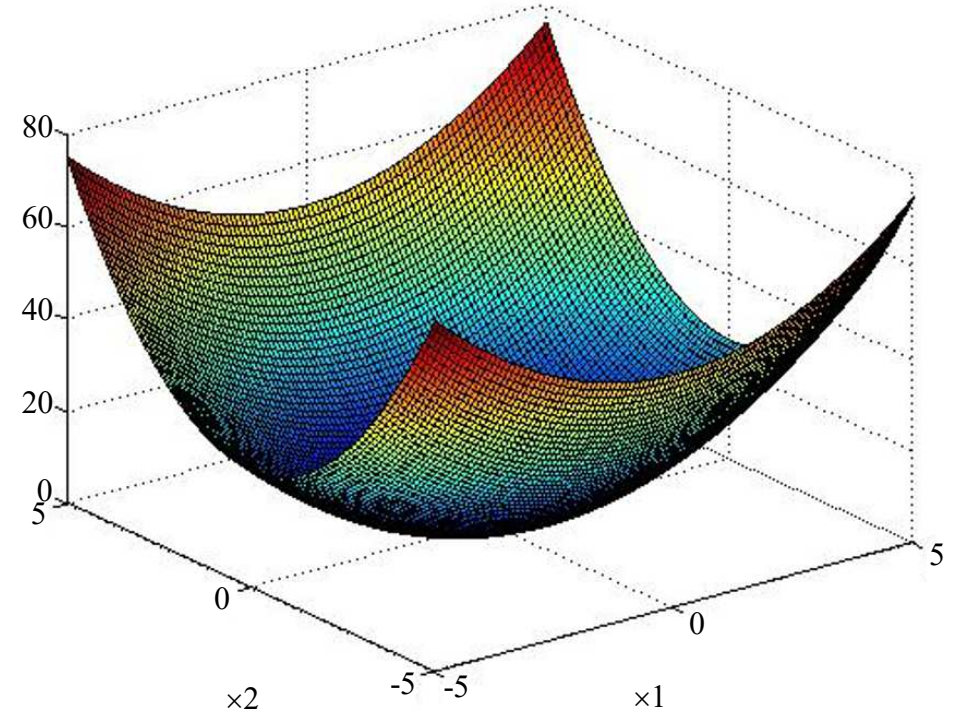

Fig. 4: Perturbed Quadratic function in 3D

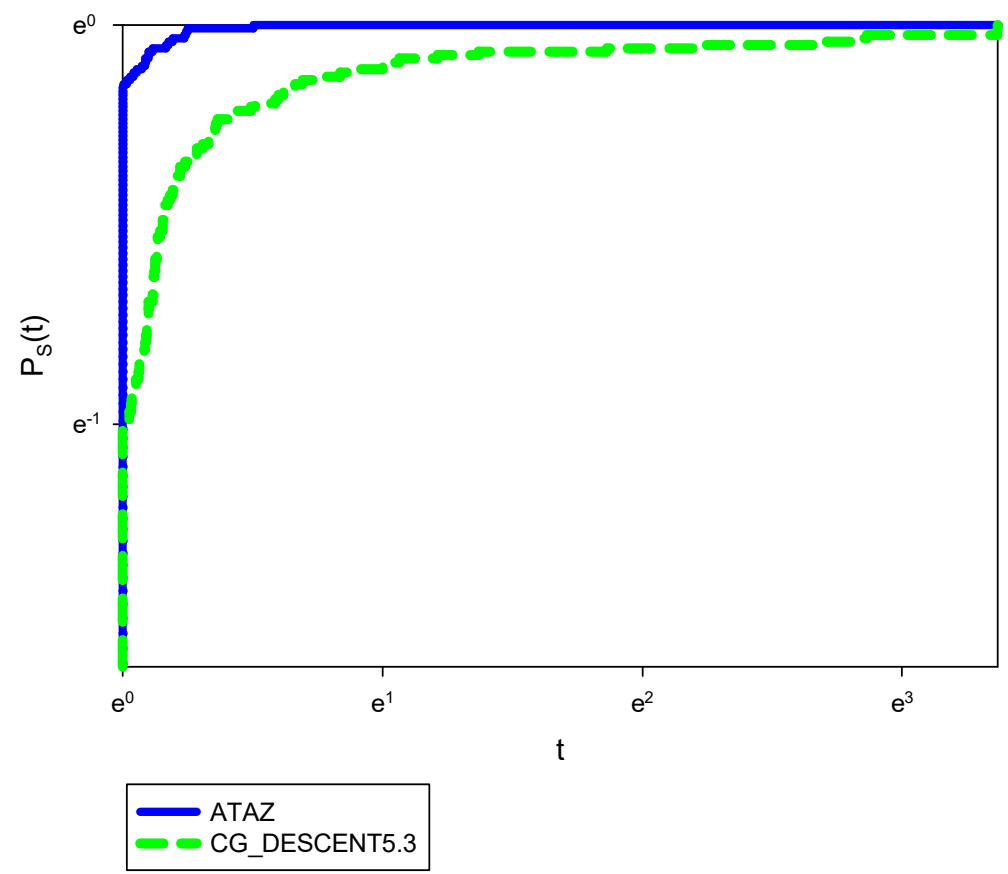

Fig. 5: Performance profile based on the number of iteration 
Ibtisam A. Masmali et al. / Journal of Computer Science 2021, 17 (6): 598.609 DOI: $10.3844 /$ jessp.2021.598.609

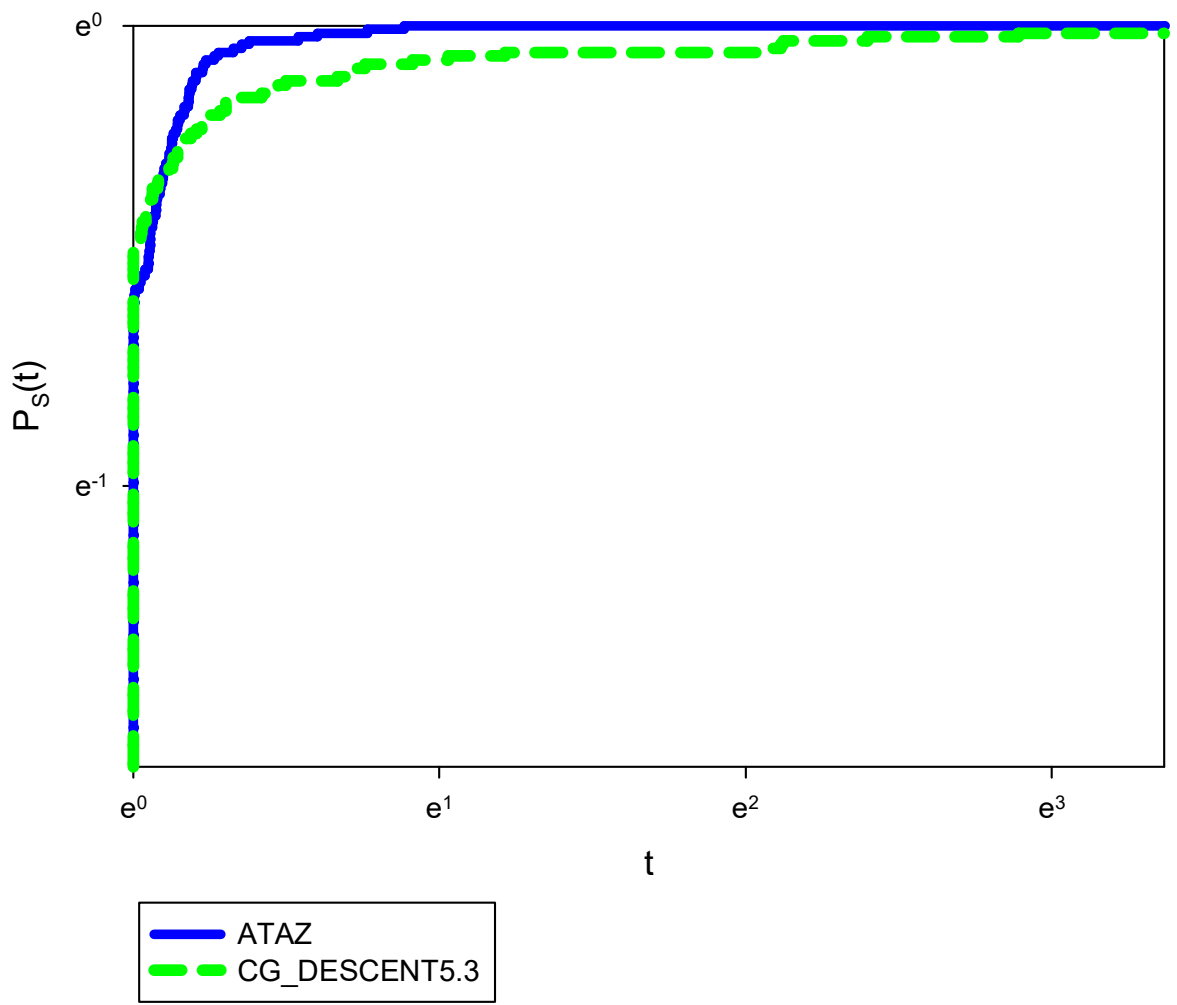

Fig. 6: Performance profile based on the function evaluation

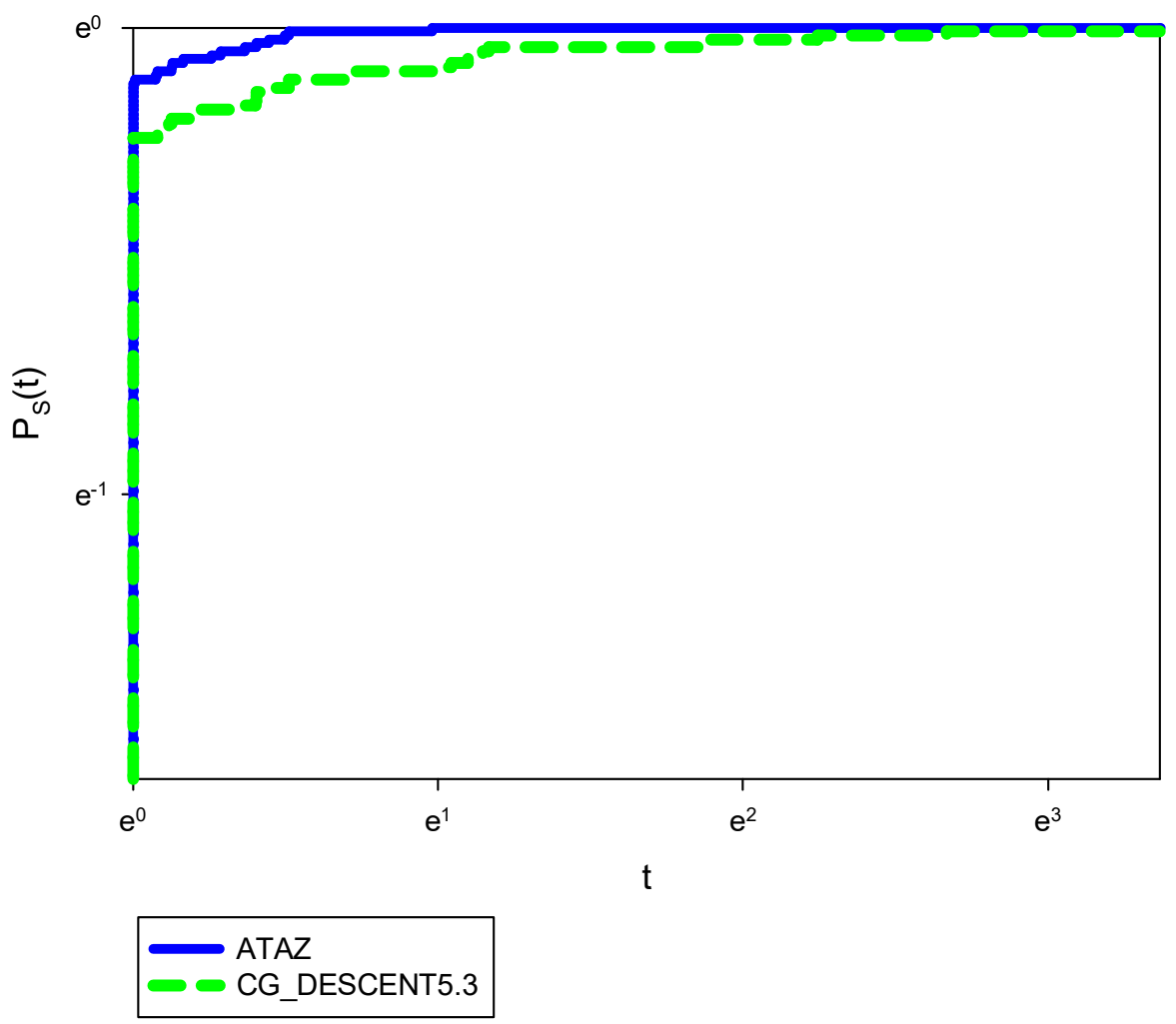

Fig. 7: Performance profile based on CPU time 
Ibtisam A. Masmali et al. / Journal of Computer Science 2021, 17 (6): 598.609 DOI: $10.3844 /$ jessp.2021.598.609

Table 2: A list of problem functions

\begin{tabular}{|c|c|c|c|c|c|c|c|}
\hline \multirow[b]{2}{*}{ function } & \multirow[b]{2}{*}{ Dimension } & \multicolumn{3}{|l|}{ ATAZ } & \multicolumn{3}{|l|}{ CG-Descent } \\
\hline & & No. iteration & $\begin{array}{l}\text { No. function } \\
\text { evaluation }\end{array}$ & CPU time & No. iteration & $\begin{array}{l}\text { No. function } \\
\text { evaluation }\end{array}$ & CPU time \\
\hline AKIVA & 2 & 8 & 20 & 0.02 & 10 & 21 & 0.02 \\
\hline ALLINITU & 4 & 9 & 25 & 0.02 & 12 & 29 & 0.02 \\
\hline ARGLINA & 200 & 1 & 3 & 0.02 & 1 & 3 & 0.02 \\
\hline ARWHEAD & 200 & 6 & 17 & 0.02 & 7 & 15 & 0.02 \\
\hline BARD & 3 & 12 & 32 & 0.02 & 16 & 33 & 0.02 \\
\hline BEALE & 2 & 11 & 33 & 0.02 & 15 & 31 & 0.02 \\
\hline BIGGS6 & 6 & 24 & 64 & 0.02 & 27 & 57 & 0.02 \\
\hline BOX3 & 3 & 10 & 23 & 0.02 & 11 & 24 & 0.02 \\
\hline BRKMCC & 2 & 5 & 11 & 0.02 & 5 & 11 & 0.02 \\
\hline BROWNAL & 200 & 9 & 22 & 0.02 & 9 & 25 & 0.02 \\
\hline BROWNBS & 2 & 10 & 24 & 0.02 & 13 & 26 & 0.02 \\
\hline BROWNDEN & 4 & 16 & 38 & 0.02 & 16 & 31 & 0.02 \\
\hline CHAINWOO & 4000 & 352 & 682 & 0.8 & 318 & 619 & 0.866 \\
\hline CHNROSNB & 50 & 269 & 549 & 0.02 & 287 & 564 & 0.02 \\
\hline CLIFF & 2 & 10 & 46 & 0.02 & 18 & 70 & 0.02 \\
\hline CUBE & 2 & 17 & 48 & 0.02 & 32 & 77 & 0.02 \\
\hline CURLY10 & 10000 & 52849 & 72728 & 197 & 47808 & 67294 & 173.7 \\
\hline CURLY20 & 10000 & 79446 & 102981 & 437 & 66587 & 89245 & 383.94 \\
\hline CURLY30 & 10000 & 81281 & 104558 & 644 & 79030 & 102516 & 639.63 \\
\hline DECONVU & 63 & 390 & 806 & $2.00 \mathrm{E}-02$ & 400 & 801 & $2.00 \mathrm{E}-02$ \\
\hline DENSCHNA & 2 & 6 & 16 & 0.02 & 9 & 19 & 0.02 \\
\hline DENSCHNB & 2 & 6 & 18 & 0.02 & 7 & 15 & 0.02 \\
\hline DENSCHNC & 2 & 11 & 36 & 0.02 & 12 & 26 & 0.02 \\
\hline DENSCHND & 3 & 14 & 46 & 0.02 & 47 & 98 & 0.02 \\
\hline DENSCHNE & 3 & 12 & 43 & 0.02 & 18 & 49 & 0.02 \\
\hline DENSCHNF & 2 & 9 & 31 & 0.02 & 8 & 17 & 0.02 \\
\hline DIXMAANA & 3000 & 6 & 16 & 0.02 & 7 & 15 & 0.02 \\
\hline DIXMAANB & 3000 & 6 & 15 & 0.02 & 6 & 13 & 0.02 \\
\hline DIXMAANC & 3000 & 6 & 14 & 0.02 & 6 & 13 & 0.02 \\
\hline DIXMAAND & 3000 & 6 & 15 & 0.02 & 7 & 15 & 0.02 \\
\hline DIXMAANE & 3000 & 13 & 31 & 0.23 & 222 & 239 & 0.33 \\
\hline DIXMAANF & 3000 & 17 & 39 & 0.02 & 161 & 323 & 0.13 \\
\hline DIXMAANH & 3000 & 44 & 103 & 0.08 & 173 & 347 & 0.22 \\
\hline DIXMAANJ & 3000 & 415 & 494 & 0.52 & 327 & 655 & 0.36 \\
\hline DIXON3DQ & 10000 & 10000 & 10007 & 19.12 & 10000 & 10007 & 19.12 \\
\hline DJTL & 2 & 75 & 1163 & 0.02 & 82 & 917 & 0.02 \\
\hline DQDRTIC & 5000 & 5 & 11 & 0.02 & 5 & 11 & 0.02 \\
\hline DQRTIC & 5000 & 15 & 32 & 0.01 & 17 & 37 & 0.03 \\
\hline EDENSCH & 2000 & 26 & 59 & 0.08 & 26 & 52 & 0.03 \\
\hline EG2 & 1000 & 3 & 13 & 0.02 & 5 & 11 & 0.02 \\
\hline EIGENALS & 2550 & 9111 & 16048 & 164 & 10083 & 18020 & 178.36 \\
\hline ENGVAL1 & 5000 & 24 & 47 & 0.08 & 27 & 50 & 0.06 \\
\hline ENGVAL2 & 3 & 26 & 73 & 0.02 & 26 & 61 & 0.02 \\
\hline EXPFIT & 2 & 9 & 29 & 0.02 & 13 & 29 & 0.02 \\
\hline EXTROSNB & 1000 & 23 & 71 & 0.8 & 3808 & 7759 & 1.25 \\
\hline FLETCBV2 & 5000 & 1 & 1 & 0.02 & 1 & 1 & 0.02 \\
\hline FLETCHCR & 1000 & 252 & 497 & 0.03 & 152 & 290 & 0.05 \\
\hline FMINSRF2 & 5625 & 23 & 83 & $1.70 \mathrm{E}-01$ & 346 & 693 & $1.09 \mathrm{E}+00$ \\
\hline FMINSURF & 5625 & 27 & 86 & 0.16 & 473 & 947 & 1.51 \\
\hline GENHUMPS & 5000 & 5740 & 15736 & 26 & 6475 & 12964 & 20.11 \\
\hline GENROSE & 500 & 1376 & 2820 & 0.28 & 1078 & 2167 & 0.17 \\
\hline GROWTHLS & 3 & 109 & 431 & 0.02 & 156 & 456 & 0.02 \\
\hline GULF & 3 & 33 & 95 & 0.02 & 37 & 84 & 0.02 \\
\hline HAIRY & 2 & 17 & 82 & 0.02 & 36 & 99 & 0.02 \\
\hline HATFLDD & 3 & 17 & 49 & 0.02 & 20 & 43 & 0.02 \\
\hline HATFLDE & 3 & 13 & 37 & 0.02 & 30 & 72 & 0.02 \\
\hline HATFLDFL & 3 & 21 & 68 & 0.02 & 39 & 92 & 0.02 \\
\hline HEART6LS & 6 & 375 & 1137 & 0.02 & 684 & 1576 & 0.02 \\
\hline HEART8LS & 8 & 253 & 657 & 0.02 & 249 & 524 & 0.02 \\
\hline HELIX & 3 & 23 & 60 & 0.02 & 23 & 49 & 0.02 \\
\hline HIELOW & 3 & 13 & 30 & 0.03 & 14 & 30 & 0.02 \\
\hline HILBERTA & 2 & 2 & 5 & 0.02 & 2 & 5 & 0.02 \\
\hline HILBERTB & 10 & 4 & 9 & 0.02 & 4 & 9 & 0.02 \\
\hline
\end{tabular}


Ibtisam A. Masmali et al. / Journal of Computer Science 2021, 17 (6): 598.609

DOI: $10.3844 /$ jessp.2021.598.609

\begin{tabular}{|c|c|c|c|c|c|c|c|}
\hline HIMMELBB & 2 & 4 & 18 & 0.02 & 10 & 28 & 0.02 \\
\hline HIMMELBF & 4 & 23 & 59 & 0.02 & 26 & 60 & 0.02 \\
\hline HIMMELBG & 2 & 7 & 22 & 0.02 & 8 & 20 & 0.02 \\
\hline HIMMELBH & 2 & 5 & 13 & 0.02 & 7 & 16 & 0.02 \\
\hline HUMPS & 2 & 45 & 223 & 0.02 & 52 & 186 & 0.02 \\
\hline JENSMP & 2 & 12 & 47 & 0.02 & 15 & 33 & 0.02 \\
\hline KOWOSB & 4 & 16 & 46 & 0.02 & 17 & 39 & 0.02 \\
\hline LIARWHD & 5000 & 19 & 50 & 0.05 & 21 & 45 & 0.03 \\
\hline LOGHAIRY & 2 & 26 & 196 & 0.02 & 27 & 81 & 0.02 \\
\hline MANCINO & 100 & 10 & 21 & 0.08 & 11 & 23 & 0.08 \\
\hline MARATOSB & 2 & 589 & 2885 & 0.02 & 1145 & 3657 & 0.02 \\
\hline MEXHAT & 2 & 14 & 59 & 0.02 & 20 & 56 & 0.02 \\
\hline MOREBV & 5000 & 25 & 102 & 0.13 & 161 & 168 & 0.41 \\
\hline NCB20B & 500 & 2044 & 2288 & 31 & 2035 & 4694 & 46.36 \\
\hline NCB20 & 5010 & 303 & 747 & 4.17 & 879 & 1511 & 11.83 \\
\hline NONCVXU2 & 5000 & 43 & 81 & 0.17 & 6610 & 12833 & 15.89 \\
\hline NONDIA & 5000 & 7 & 25 & 0.02 & 7 & 25 & 0.03 \\
\hline NONDQUAR & 5000 & 66 & 216 & 0.17 & 1942 & 3888 & 2.45 \\
\hline OSBORNEA & 5 & 82 & 230 & 0.02 & 94 & 213 & 0.02 \\
\hline OSBORNEB & 11 & 57 & 134 & 0.02 & 62 & 127 & 0.02 \\
\hline OSCIPATH & 10 & 295029 & 781729 & 2.24 & 310990 & 670953 & 2.08 \\
\hline PALMER1C & 8 & 12 & 27 & 0.02 & 11 & 26 & 0.02 \\
\hline PALMER1D & 7 & 10 & 24 & 0.02 & 11 & 25 & 0.02 \\
\hline PALMER2C & 8 & 11 & 21 & 0.02 & 11 & 21 & 0.02 \\
\hline PALMER3C & 8 & 11 & 21 & 0.02 & 11 & 20 & 0.02 \\
\hline PALMER4C & 8 & 11 & 21 & 0.02 & 11 & 20 & 0.02 \\
\hline PALMER5C & 6 & 6 & 13 & 0.02 & 6 & 13 & 0.02 \\
\hline PALMER6C & 8 & 11 & 24 & 0.02 & 11 & 24 & 0.02 \\
\hline PALMER7C & 8 & 11 & 20 & 0.02 & 11 & 20 & 0.02 \\
\hline PALMER8C & 8 & 11 & 19 & 0.02 & 11 & 18 & 0.02 \\
\hline PENALTY1 & 1000 & 14 & 51 & 0.02 & 28 & 69 & 0.02 \\
\hline PENALTY2 & 200 & 202 & 238 & 0.03 & 191 & 221 & 0.05 \\
\hline PENALTY3 & 200 & 36 & 102 & 0.58 & 99 & 285 & 1.78 \\
\hline POWELLSG & 5000 & 28 & 70 & 0.01 & 26 & 53 & 0.02 \\
\hline POWER & 10000 & 360 & 736 & 0.63 & 372 & 754 & 0.76 \\
\hline QUARTC & 5000 & 15 & 32 & 0.02 & 17 & 37 & 0.03 \\
\hline ROSENBR & 2 & 28 & 84 & 0.02 & 34 & 77 & 0.02 \\
\hline S308 & 2 & 7 & 21 & 0.02 & 8 & 19 & 0.02 \\
\hline SCHMVETT & 5000 & 41 & 72 & 0.19 & 43 & 73 & 0.23 \\
\hline SENSORS & 100 & 27 & 73 & 0.39 & 21 & 50 & 0.25 \\
\hline SINEVAL & 2 & 46 & 181 & 0.02 & 64 & 144 & 0.02 \\
\hline SINQUAD & 5000 & 14 & 45 & 0.08 & 14 & 40 & 0.09 \\
\hline SISSER & 2 & 5 & 19 & 0.02 & 6 & 18 & 0.02 \\
\hline SNAIL & 2 & 61 & 251 & 0.02 & 100 & 230 & 0.02 \\
\hline SPARSINE & 5000 & 22306 & 22552 & 86 & 18358 & 18647 & 73 \\
\hline SROSENBR & 5000 & 9 & 23 & 0.02 & 11 & 23 & 0.02 \\
\hline STRATEC & 10 & 170 & 419 & 6.22 & 462 & 1043 & 19.98 \\
\hline TESTQUAD & 5000 & 1580 & 1587 & $1.34 \mathrm{E}+00$ & 1577 & 1584 & $1.52 \mathrm{E}+00$ \\
\hline TOINTGOR & 50 & 118 & 215 & 0.02 & 135 & 233 & 0.02 \\
\hline TOINTGSS & 5000 & 4 & 9 & 0.02 & 4 & 9 & 0.02 \\
\hline TOINTPSP & 50 & 120 & 262 & 0.02 & 143 & 279 & 0.02 \\
\hline TOINTQOR & 50 & 29 & 36 & 0.02 & 29 & 36 & 0.02 \\
\hline TQUARTIC & 5000 & 11 & 41 & 0.03 & 14 & 40 & 0.03 \\
\hline TRIDIA & 5000 & 783 & 790 & 0.91 & 782 & 789 & 0.84 \\
\hline VARDIM & 200 & 9 & 20 & 0.02 & 11 & 25 & 0.02 \\
\hline VAREIGVL & 50 & 24 & 54 & 0.02 & 23 & 47 & 0.02 \\
\hline VIBRBEAM & 8 & 98 & 255 & 0.02 & 138 & 323 & 0.02 \\
\hline WATSON & 12 & 58 & 219 & 0.02 & 49 & 102 & 0.02 \\
\hline WOODS & 4000 & 24 & 61 & 0.03 & 22 & 51 & 0.06 \\
\hline YFITU & 3 & 68 & 208 & 0.02 & 84 & 197 & 0.02 \\
\hline ZANGWIL2 & 2 & 1 & 3 & 0.02 & 1 & 3 & 0.02 \\
\hline
\end{tabular}




\section{Conclusion}

In this study, we proposed a new modification of conjugate gradient method $\left(d_{k}^{A T A Z}\right)$ with restart condition is presented. In addition, new restart criterion proposed for FR CG method. Our numerical results had shown that the new coefficients are better than other conventional CG methods. Furthermore the efficiency of the FR method improved substantially when the new restart criterion is used.

\section{Acknowledgements}

The authors are grateful to the editor and the anonymous reviewers for their valuable comments and suggestions, which have substantially improved this paper. This research was partially supported by the Center of Research and Innovation Management, Universiti Malaysia Terengganu.

\section{Author's Contributions}

Ahmad Alhawarat: Methodology and sofrware.

Zabidin Salleh: Proof theory and review the paper with the software.

Ibtisam A. Masmali: Support software and review the main CG formula with its proof.

\section{Ethics}

This article is original and contains unpublished material. The corresponding author confirms that all of the other authors have read and approved the manuscript and no ethical issues involved.

\section{References}

Al-Baali, M. (1985). Descent property and global convergence of the Fletcher-Reeves method with inexact line search. IMA Journal of Numerical Analysis, 5(1), 121-124. https://doi.org/10.1093/imanum/5.1.121

Alhawarat, A., Salleh, Z., Mamat, M., \& Rivaie, M. (2017). An efficient modified Polak-Ribière-Polyak conjugate gradient method with global convergence properties. Optimization Methods and Software, 32(6), 1299-1312. https://doi.org/10.1080/10556788.2016.1266354

Andrei, N. (2008). An unconstrained optimization test functions collection. Advance Modelling Optima, 10(1), 147-161.

Beale, E. M. L. (1972). A deviation of conjugate gradients. Numerical Methods for Nonlinear Optimization, 39-43.

Bongartz, I., Conn, A. R., Gould, N., \& Toint, P. L., (1995). CUTE: Constrained and unconstrained testing environment. ACM Transactions on Mathematical Software (TOMS), 21(1), pp, 123-160. https://doi.org/10.1145/200979.201043
Dai, Y. H., \& Liao, L. Z. (2001). New conjugacy conditions and related nonlinear conjugate gradient methods. Applied Mathematics and Optimization, 43(1), 87-101. https://doi.org/10.1007/s002450010019

Dai, Y., \& Yuan, Y. (1998). Convergence properties of Beale-Powell restart algorithm. Science in China Series A: Mathematics, 41(11), 1142-1150. https://doi.org/10.1007/BF02871976

Dolan, E. D., \& Moré, J. J. (2002). Benchmarking optimization software with performance profiles. Mathematical Programming, 91(2), 201-213. https://doi.org/10.1007/s101070100263

Fletcher, R., \& Reeves, C. M. (1964). Function minimization by conjugate gradients. The Computer Journal, 7(2), 149-154. https://doi.org/10.1093/comjnl/7.2.149

Gilbert, J. C., \& Nocedal, J. (1992). Global convergence properties of conjugate gradient methods for optimization. SIAM Journal on optimization, 2(1), 21-42. https://doi.org/10.1137/0802003

Guanghui, L., Jiye, H., \& Hongxia, Y. (1995). Global convergence of the Fletcher-Reeves algorithm with inexact linesearch. Applied Mathematics-A Journal of Chinese Universities, 10(1), 75-82. https://doi.org/10.1007/BF02663897

Hager, W. W., \& Zhang, H. (2005). A new conjugate gradient method with guaranteed descent and an efficient line search. SIAM Journal on optimization, 16(1), 170-192. https://doi.org/10.1137/030601880

Hager, W. W., \& Zhang, H. (2013). The limited memory conjugate gradient method. SIAM Journal on Optimization, 23(4), 2150-2168. https://doi.org/10.1137/120898097

Hestenes, M. R., \& Stiefel, E. (1952). Methods of conjugate gradients for solving linear systems (Vol. 49, No. 1). Washington, DC: NBS. https://doi.org/10.6028/jres.049.044

Kaelo, P., Mtagulwa, P., \& Thuto, M. V. (2020). A globally convergent hybrid conjugate gradient method with strong Wolfe conditions for unconstrained optimization. Mathematical Sciences, 14(1), 1-9. https://doi.org/10.1007/s40096-019-00310-y

Polak, E., \& Ribiere, G. (1969). Note on the convergence of conjugate direction methods. ESAIM: Mathematical Modeling and Numerical Analysis-Mathematical Modeling and Numerical Analysis, 3 (R1), 35-43. https://doi.org/10.1051/m2an/196903R100351

Powell, M. J. (1984). Nonconvex minimization calculations and the conjugate gradient method. In Numerical analysis (pp. 122-141). Springer, Berlin, Heidelberg. https://doi.org/10.1007/BFb0099521

Powell, M. J. D. (1977). Restart procedures for the conjugate gradient method. Mathematical Programming, 12(1), 241-254. https://doi.org/10.1007/BF01593790 
Ibtisam A. Masmali et al. / Journal of Computer Science 2021, 17 (6): 598.609

DOI: $10.3844 /$ jessp.2021.598.609

Wolfe, P. (1969). Convergence conditions for ascent methods. SIAM Review, 11(2), 226-235. https://doi.org/10.1137/1011036

Wolfe, P. (1971). Convergence conditions for ascent methods. II: Some corrections. SIAM Review, 13(2), 185-188. https://doi.org/10.1137/1013035
Zoutendijk, G. (1970). Nonlinear programming, computational methods. Integer and Nonlinear Programming, 37-86.

https:/ci.nii.ac.jp/naid/10030666308/ 